\title{
Milk-Run Collection Monitoring System Using the Internet of Things Based on Swarm Intelligence
}

\author{
Yassine Karouani, RITM Laboratory, Morocco \\ (iD) https://orcid.org/0000-0001-6396-2010 \\ Mouhcine Elgarej, Laboratory SSDIA, ENSET Mohammedia, Morocco
}

\begin{abstract}
In Morocco, several dairy factories are placed in rural regions with a bad road network, which means that milk collection has a significant impact on profit, affecting milk transport costs. Actually, the milk run logistics process has been transformed from a traditional farm to the new cheese factory, so it's needed efficient methods and models to improve the process of production and collection of milk from those units. For that, the authors apply new technologies such as the internet of things (IoT) and big data to collect and analyze this information to optimize the milk delivery process. The main goal of this work is to design a new smart decision method using the internet of things and big data to optimize the milk run logistics, reduce the cost of transportation, and improve collection density. This method will be based on the swarm artificial intelligence concept to find and calculate the shortest path between units to optimize the collection of milk.
\end{abstract}

\section{KEYWORDS}

Ant Colony Optimization, Artificial Intelligent, Internet of Things, Milk-Run, Multi-Agent System, Smart Transportation and Logistic, Swarm Intelligence, Vehicle Routing System

\section{INTRODUCTION}

An excellent growth medium for microorganisms, milk is a highly perishable nutritious food. Processing allows the preservation of this product for several days, as well as the extension of its shelf life. This is achieved through many techniques, including cooling or fermentation. The processing of raw milk into processed dairy (Bocewicz, $\mathrm{G}$ et $\mathrm{Al}, 2019$ ) products can help to cope with seasonal fluctuations in milk production by generating off-farm employment in the collection, transportation, processing and marketing of milk.

A Milk Run (Nguyen, et Al, 2016) is a delivery strategy used to transport milk from various farms. Instead of each farm sending a truck every day to collect milk. The arrangement of the circulation trailer tank milk collection problem is a key element to determine whether the pattern of vehicle supply logistics cycle pickup can implement success. Arrange the milk collection path reasonably is beneficial to reduce transportation cost, save time and improve the efficiency of the milk logistics operations.

\section{DOI: 10.4018/IJISSCM.290018}

This article published as an Open Access article distributed under the terms of the Creative Commons Attribution License (http://creativecommons.org/licenses/by/4.0/) which permits unrestricted use, distribution, and production in any medium, provided the author of the original work and original publication source are properly credited. 
Vehicle routing problem (Ma, J., \& Sun, G. 2013) can be described as that in a system which exists supply and demand; there are a set of vehicles and several customers, the company should visit all of those customers based on the available vehicles. Milk logistics (Caria, M et Al, 2018) factories use multiple vehicles to take milk from farms, the position of each farm and the capacity of trailer tank are constant to ensure that the total cost of the trip is the best condition. It is necessary to make reasonable path planning for vehicles and reduce the traveling time to visit all the available farms.

In the case of our country, we five types of dairy factory can be distinguished in Morocco: (i) milk collection cooperatives; (ii) livestock breeders; (iii) industrial processors of dairy products; (iv) traditional milk shops; (v) milk collectors/vendors in informal chains. Currently, three types of dairy factories are identified in our supply chain management, the first are private chains that collect milk from industrial dairy processors and farms through milk collection cooperatives. The second type of chain is based on cooperative processing and has recently seen the emergence of a very competitive cooperative. The third type of dairy chain is based on informal channels, with milk being processed into traditional products by small workshops and sold directly to consumers. These informal channels involve a variable number of operators and are mainly active in the dairy basins near the major urban centers.

The main goal of this paper is to design a new decision support tool for smart cities based on big data combined with the Internet of Things technologies 'Elgarej et al, 2020) to optimize milk path planning, improve milk collection density and reduce the cost of milk transportation. In supply chain management, IoT devices represent an effective means for collecting, tracking and the ability for sharing data with database centers which use Apache Hadoop big data technology.

The structure of this paper is organized as follow, we start by the related work section then in the second section, we describe the basic concept of the internet of things and it's majors forces, the third section, is dedicated to present the basic processing of dairy factory systems in Morocco and we introduce the standard strategy used for the transportation and the collection of milk. The fourth section, will be reserved for our proposed distributed strategy dedicated to the milk-run system based on the IoT technologies combined with multi-agent system to find and compute best paths planning. Finally, we discuss the simulation process of our new strategy based on real-life cases to analyze the results of our strategy compared with the traditional method used for the collection of milk in Morocco. We conclude our work with a general conclusion that summary our approaches, which aim to find a new distributed and efficient strategy to improve the productivity of the milk-run transportation system in Morocco.

\section{RELATED WORK}

Actually, with the need of controlling costs of milk collecting and distribution to solve the problem of the weak arrangement of routes and vehicles planning. Authors in (K. Ji-li et al, 2013) propose a new mathematical pattern based on the time window concept for the vehicle routing system. The main goal of this model is to optimize the total path crossed by vehicles for milk collection and reduce the waiting time for pick-up of milk from farms. The resulting feedback has been significant according to the data set used for running the new proposed model.

The concept of milk run has been adopted in supply chain management to control and manage the routing system based on the available transportation materials. In this paper (M. Jafari-Eskandari et al, 2009) a new method is modeled to solve the problem of milk run logistics based on a mathematical model which use the constraint of milk run as a deciding factor combined with a robust optimization technic to find the best solution. The results of the simulation have shown good solution according to the constraints of the problem used to find the best solutions.

To increase the productivity of companies we need to use some alternative solutions and smart equipment in order to face the current economic challenges. Those smart solutions or methods are used to increase the productivity and to control the cycle life of the product from construction to delivering 
for the final customers. One of those problems we have the milk run logistics, which aim to collect a set of the quantity of milk from various units and we need to find the best cost of transportation to move out this quantity to a specific central unit. In this work (Eva Klenk et al, 2015) a new intelligent milk-run system based on learning factory and using the RFID technology to control and manage the process of collection and distribution of milk. On the other hand, they use a new concept for path planning based on the data retrieved from the system to compute and find the shortest path from the main warehouse to the workstation.

The concept of using digital technologies has been adopted to improve the control and the management of industrial production life cycle. To manage this process we need to use a set of intelligent objects (things) to do the hard work and collect data from various sectors, but we need to find a good way to analyze those data based on the mechanism of big data. Here we can see the role of big data to manage and control the data shared between things using the internet. Several kinds of research have been done to get the link between the internet of things and big data and to see the advantage of combining those elements to improve the benefits of companies. As the authors said (Andrea Sestino et al, 2020), Big data and IoT are useful for improving the quality of services provided by companies.

\section{THE CONCEPT OF THE INTERNET OF THINGS}

A new paradigm called the Internet of Things has rapidly gained ground in recent years. IoT refers to "a global network of uniquely addressable interconnected objects based on standard communication protocols" (Qu, T et al, 2015) whose point of convergence is the Internet. The main consequence of IoT is, without a doubt, its impact on the daily life of potential users. IoT has remarkable effects both in the home and at work where it will play a decisive role shortly (health, intelligent transportation, home automation, assisted living, etc.). Significant spin-offs are also expected for businesses (freight transport, security, logistics, industrial automation, etc.). Based on these considerations, the U.S. National Intelligence Council has identified IoT as one of the six technologies that will have a potential impact on U.S. interests by 2025 (Zhang, H., \& Lu, X. 2020).

The Internet is gradually transforming into a HyperNetwork, as a network formed by multitudes of connections (Figure 1) between Artifacts (physical, documentary), actors (biological, algorithmic), writings and concepts (linked data, metadata, ontology), called "Internet of Things (IoT) Internet of Things (IoT)", connecting billions of human beings but also billions of objects. It is becoming the most powerful tool ever invented by man to create, modify and share information. This transformation shows the evolution of the Internet network: from a network of computers to a network of personal computers, then to a nomadic network integrating communication technologies (Kothandaraman, D, et al, 2019). Developments in Machine-to-Machine (M2M) technologies for remote machine control and also the appearance in the year 2000 of IP (Internet Protocol) on mobile cellular networks have accelerated the evolution of M2M towards IoT.

Connected objects produce large amounts of data, the storage and processing of which is called big data. In logistics, these can be sensors that are used to trace goods for inventory management and routing. In the environment, it is a question of sensors monitoring air quality, temperature, noise level, the state of a building, etc. In home automation, IoT covers all communicating household appliances, sensors (thermostat, smoke detectors, presence detectors, etc.), smart meters and security systems connected to home automation box-type devices.

\section{DAIRY INDUSTRY AND TRANSPORT MANAGMENT}

The dairy sector represents one of the rare agricultural activities where the producer price is stable and known and where the outlets are assured compared to other speculations (market gardening, tree-culture, etc.). This stability is an undeniable attraction for many economic actors, particularly 


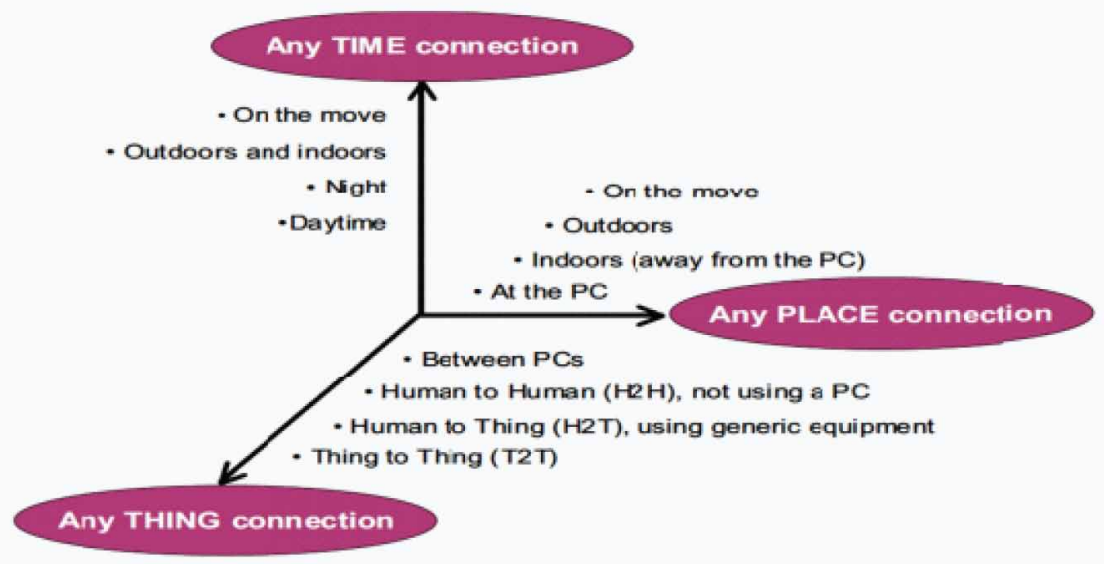

breeders. However, with the re-bidding of concentrated feeds, producers must strive to produce at a cost price lower than the selling price of milk. As for imports, they reach high levels during the Ramadan period when the consumption of milk and dairy products increases sharply. They are particularly high for butter, powdered milk, and cheese.

The Milk-Run (Kale S, 2020) process is used in logistics to design a vehicle tour, which tries to visit a set of customers to deliver and pick-up of milk bottles. The Milk-Run (Kong, J. L et al, 2013) system was largely adopted in business-to-business transport and was combined with the new technology systems to improve the quality of services provided by this domain. Modelling the logistics of dairy collection and delivery is an essential feature of improving the profitability of the system and decrease the carbon emissions during the process of collection and travelling.

A milk collection network (Figure 2) consists of a number of milk collection centers located at shipping points and plants. A fleet of vehicles, consisting of vehicles of different capacities and with different fixed and variable costs, is available at the shipping points. The volume of milk at the collection centers varies from day to day and varies according to the season. However, the supply for a given day is known before the vehicles leave the dispatch point. Vehicles collect milk daily from the collection centers and transport it to the respective shipping points. All milk must be collected at each milk collection center. All vehicles must complete their rounds within a specified time frame. We use distance as an approximation of time and introduce a distance-time constraint into our formulation. The problem faced by dairy processors (Minh, et al, 2020) is essentially related to collecting all available milk at the lowest possible cost. Milk collection costs include the costs of operating the shipping center and the costs of transporting vehicles and constitute a significant portion of the total cost of milk, the other important cost being the cost of the milk itself.

Our objective is to find the number and location of collection points, the number of each type of vehicle and the vehicle routes that would minimize transportation costs that include fixed vehicle costs and mileage costs. The constraints are:

- The total supply from all supply points on a given route must not exceed the capacity of the vehicle assigned to that route.

- Each collection point must be visited by a single vehicle.

- Each vehicle makes a single trip and begins and ends at the collection center or central shipping point.

- The vehicle must complete its journey on a route within a specified time. 


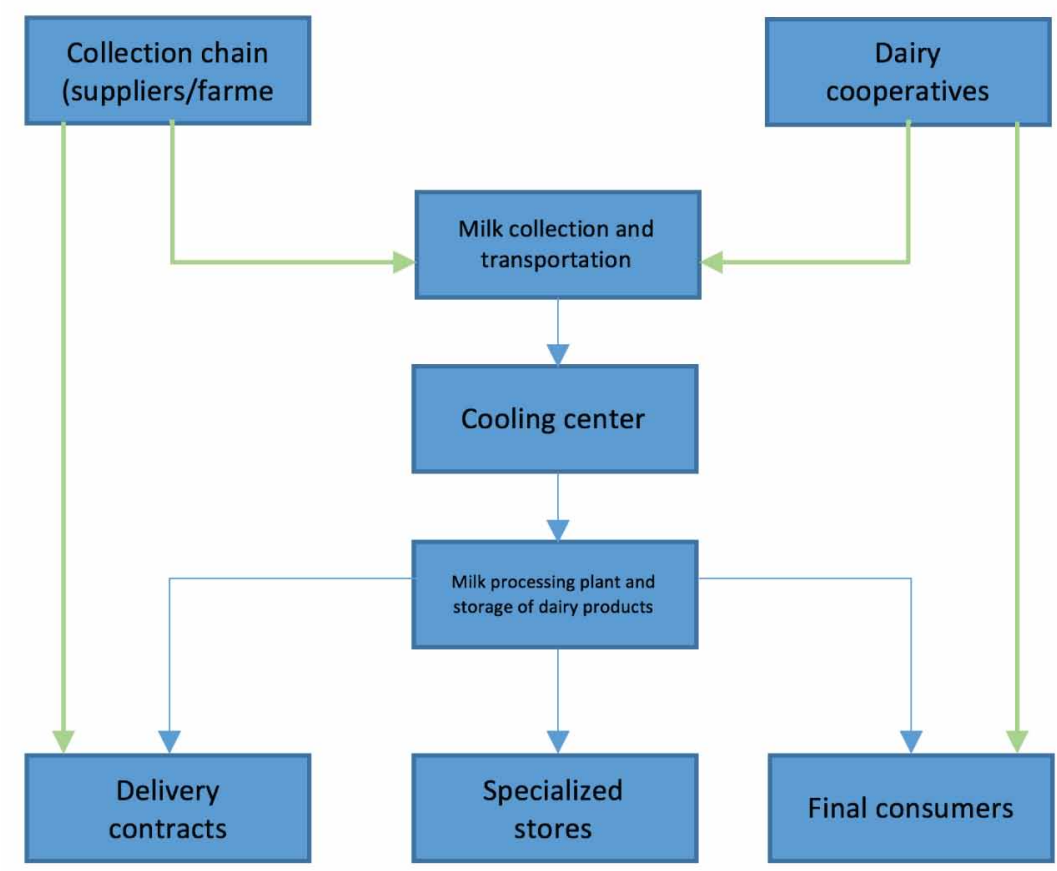

Our objective is to improve the milk collection process using the new alternatives strategies and technologies to transform the milk run process from traditional methods based on the regular or periodic visit of a set of farms for picking up milk, but in this method the driver has no idea about the quantity of milk to pick-up and they have no information about the shortest path that should take to finish their daily tour. To improve this process, we will try to introduce the concept of IoT to prepare the environment and collect data that will be used by our routing system that use the swarm intelligence behavior to prepare the best path planning that will be followed by the driver to visit the distributed farms. Our objective will be:

- Produce a path planning for each truck, this path will visit a set of farms units, we will take the time allowed for the collection, the maximum capacity of the vehicle and number of farms to visit per day to compute this path.

- Share with the controlled a set of information about the quantity of milk available at each unit, so we will be able to have visibility about the quantity of milk that will be shipped.

\section{IMPLEMENTATION OF A DISTRIBUTED STRATEGY FOR THE MILK-RUN BASED ON THE INTERNET OF THINGS}

Our main approach is to make a new decision model based (Chen, Z., \& Sarker, B. R. 2014) on the data collected from smart farms such as the geographic location of the farm and the available quantity of milk. That information will be used in our model to produce a set of paths planning for the process of milk collection. In this model, we will try to search for the shortest path between the visiting farms, also, we need to minimize the number of reserved trucks used for milk collection. The 
result of our approach is to give you the best shortest path that visit a set of farms with the minimum number of trucks for milk collection.

Our architecture will consist of a set of distributed components (Elgarej et al, 2019) that are connected through the Internet (Figure 3). The data shared during this process of communication will be stored in a special database based on MongoDB. This type of database will help us to store huge data and will allow us to retrieve and request data in a few milliseconds. To observe the process of collection and transportation, we created a third application (based on mobile and web) that allows us to control the process of milk transportation and to supervise the life cycle trips done by each truck.

\section{Development of the Distributed Model for Milk Transport}

For the implementation of our milk collection and control model (Hfeda, M, et al, 2017), we have opted for a distributed solution (Figure 4) based on agents interconnected via asynchronous messages. The

Figure 3. A distributed architecture for milk colelction system

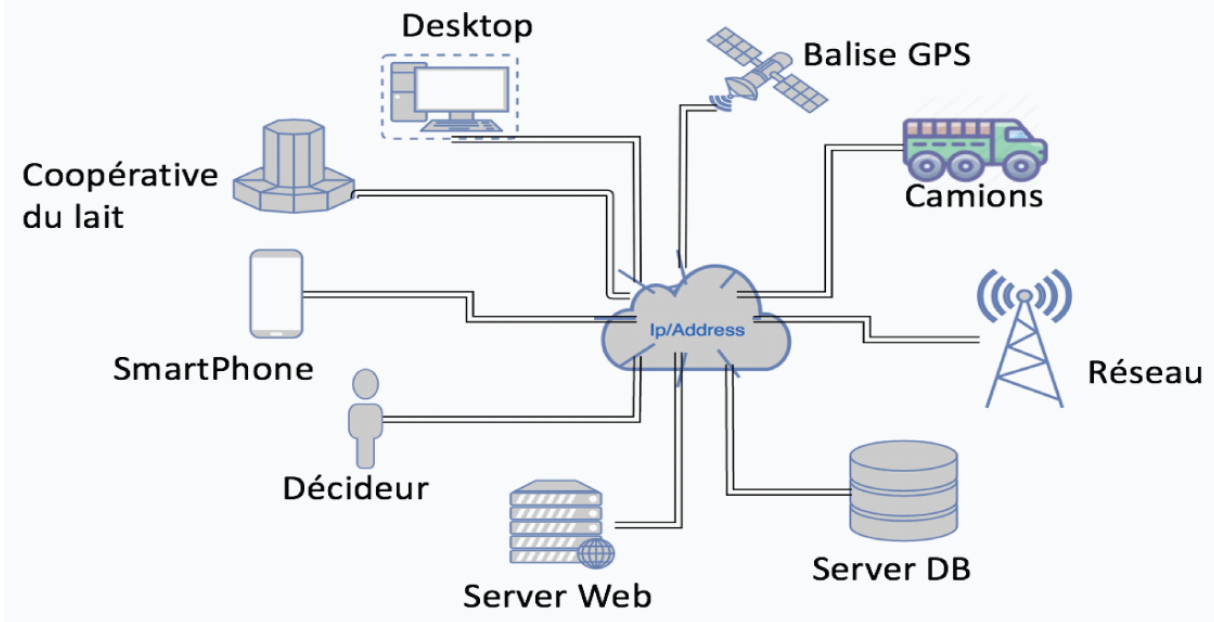

Figure 4. Multi-agent strategy for Milk-run collection system

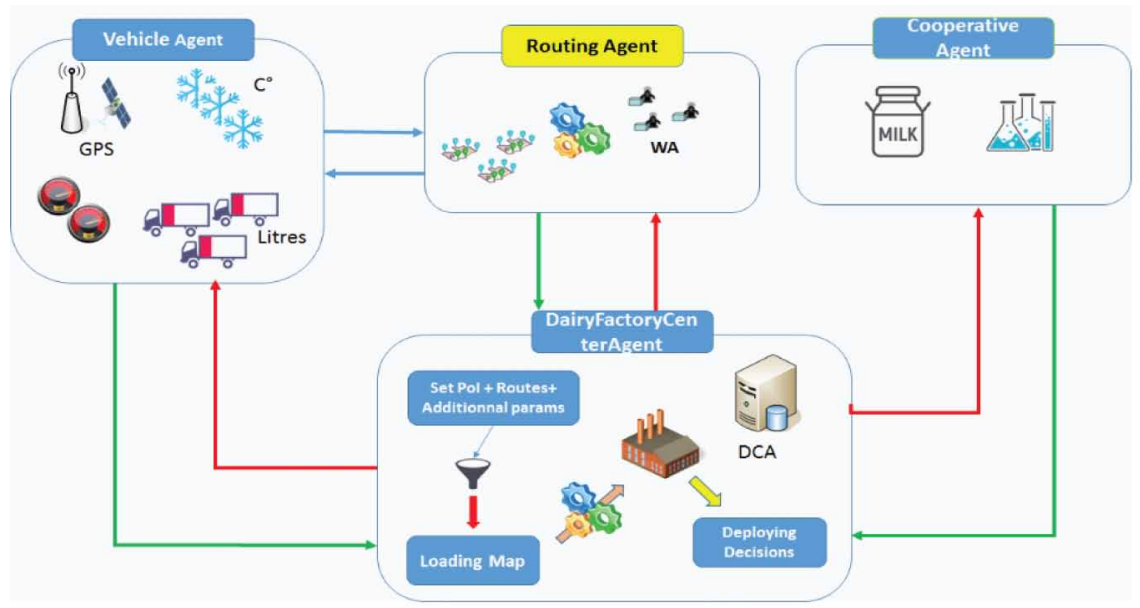


objective of this agent-based approach is to make our system evolve, as well as to separate the process of control and calculation of the best paths, and the process of supervision and decision support. As you can see, our architecture consists of agents subdivided into three categories:

- Data collection and formalization category: This range of agents takes care of the collection and factorization of data to prepare the environment and the search space that will be subsequently adopted for the constitution of the best paths.

- Routing category: This category gathers agents that will use ant colony optimization techniques to search for the best paths in terms of travel distance and total quantity of milk collected and finally in terms of travel time and collection.

- Monitoring and supervision category: Its agents are designed to prepare data and information that will be projected for the supervisor and the user of our solution, namely activity reports and traffic states as well as reports on delays and anomalies experienced during the milk collection process.

\section{Cooperative Agent}

For our case, we assume that each farm inside our system will be controlled by an agent, the main goal of this one is:

- To share the actual latitude and longitude of this farm with the remote system;

- To share in real-time, the current quantity of milk inside this cooperative, for this total quantity, this agent will be able to give us the quality of the collected milk (in liters) based on the information retrieved from the testing machine, so this total quantity will be split into three main categories:

- Green: The quality of the collected milk is good;

- Red: The quality of the collected milk is bad;

- Yellow: The quality of the collected milk is medium.

The testing machine will give us a vision on the quantity of milk that we will collect from this cooperative. Using that information, we will be able to minimize the number of the truck that will visit this cooperative also the supervisor can ignore this unity in his path planning. So as you can see, this agent will give us a set of information that will be used by the DFCA in the process of creating the set of path planning for a specific area.

A cooperative is viewed as a collection point that will be visited by a truck. All milk units will be shown inside a geographical map (figure 5) that will be converted to a complete graph which will be implemented by the DFCA for the process of computing and finding the best paths to visited each unit. Those units will be clustered inside a set of sub-areas. This approach will help us to reduce the size of the global space of search and also will help us to control and monitor each area with his milk units.

\section{Vehicle Agent}

As presented in the previous architecture, we need to supervise the path followed by a truck. to do that, we assume that each truck inside our model will be tracked based on a GPS component on board. The information that will be produced by this component are (figure 6): the real-time location of the truck, the current speed, the fuel consumed during the trip, the temperature of the engine, the temperature of the tank that contains the collected milk. As you know, our main goal is to keep our tank at the standard level of cooling which is between $-10^{\circ}$ to $12^{\circ}$. finally, this component gives us the quantity of milk inside the tank.

In our case, each truck will be controlled by a vehicle agent (VA), this one will keep an eye on the status of the truck based on the GPS component, and will share all of the information received from the GPS with the FDCA based on the cellular data 5G. When a new path planning is computed and assigned to this truck, this agent will be able to show this path for the driver and to help them in 
Figure 5. Geographical map with a set of milk units

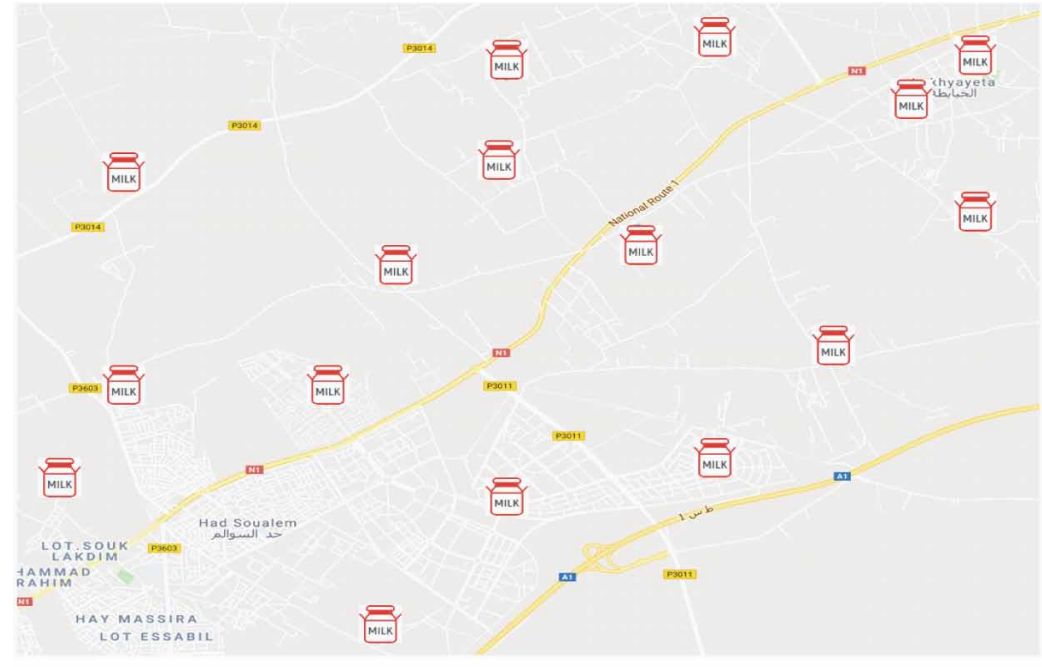

Figure 6. Dashboard of truck data provided by the GPS

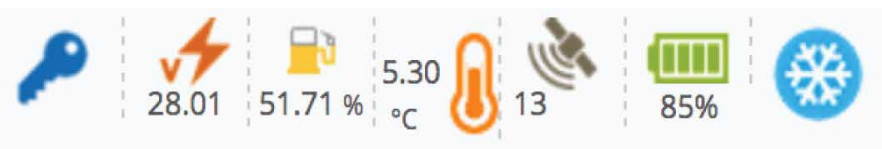

the process of collection by giving them all the information needed to complete his journey such as the number of units of milk to visit, the total distance to travel, the estimated time to complete the journey based on the real-time of traffic flow, finally, the total quantity of milk that will be collected. After each visited milk unit, the VA will update his trip and send the DFCA the last information about his trip.

\section{Dairy Factory Center Agent}

The proposed architecture is based on a distributed strategy in which we have a set of autonomous agents, they work in parallel to collect and compute the best solutions in the minimum time. The DFCA agent is viewed as the main controller inside our architecture, the role of this agent is divided into two layers:

- Collection and environment construction: To make our dairy milk collection network we need to provide a mechanism that will be able to collect all information about milk units and set them inside a graph. This graph will be made from a set of locations (milk unit) and routes between those locations (figure 7), for the cost of these routes will be characterized by the distance in kilometers and the traffic flow. To set the milk units, we need to contact the CAs to gives us the set of GPS locations, those data will be injected into our graph. After the collection network is created, a new step is started to create sub-graphs from the global graph, this process is done based on a clustering system. The clustering strategy allows us to reduce the complexity of our problems and to create a small area which are made from the closets milk units. In our case, we 
Figure 7. An overview of the real-time location of a truck

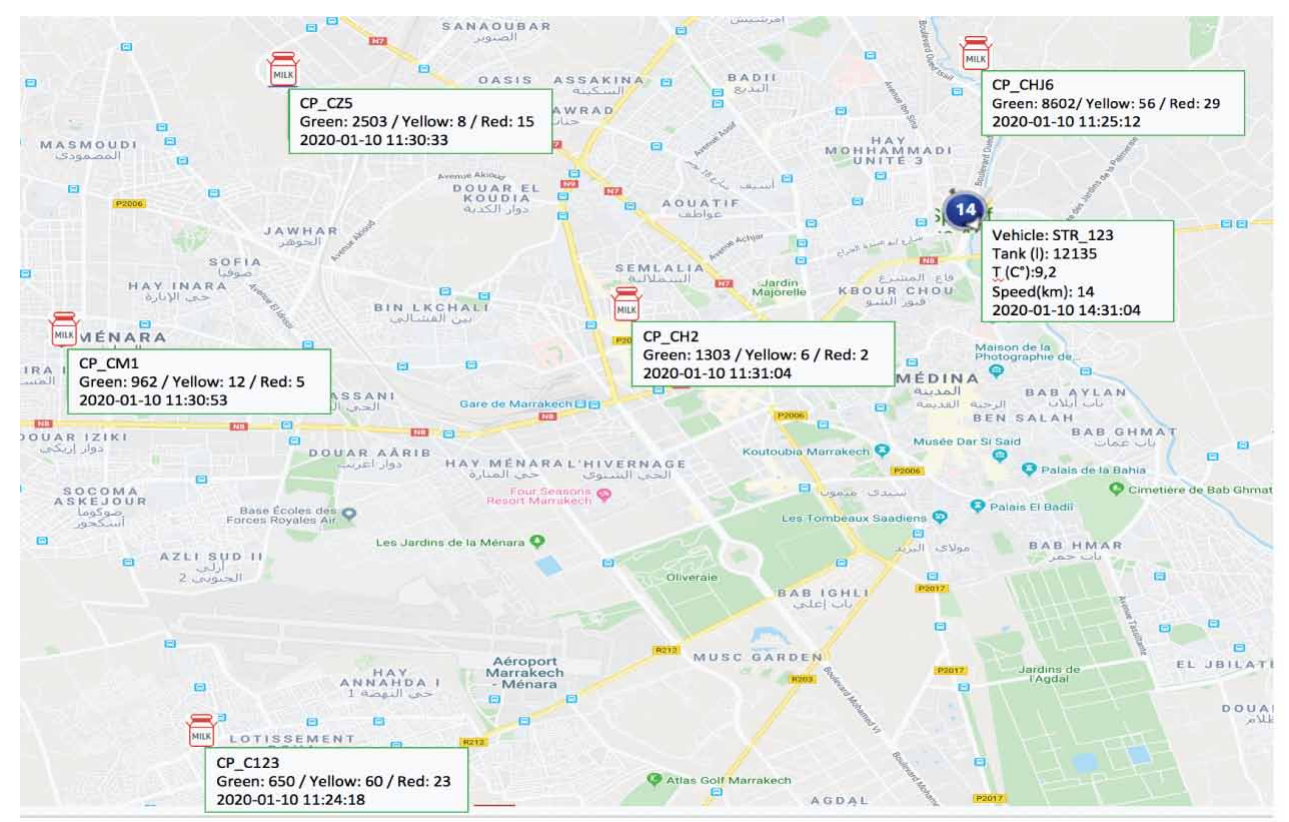

apply the K-means Clustering algorithm to generate those sub-areas based on the linear distance between the set of nodes. The result of this clustering will allow us to have visibility about the number of nodes to visit inside each area, also, we have an idea about the total distance that will be covered by a truck and the total quantity of milk to pick up from those cooperatives. In our case, we suppose that each vehicle is characterized by its maximum capacity that he should not exceed during the process of collection, so when the supervisor tries to link each vehicle to a sub-area, the system will give him a set of notifications if the total quantity of milk inside this area is higher than the capacity of the truck.

- Path planning distribution: After all sub-graphs are made, the DFCA will share those areas with the RA, this one will take those data and start the process of computing the best path planning for each sub-area according to the distributed ant colony optimization strategy (Starzec, $\mathrm{M}$ et al, 2020). The result of this transaction will be returned to the DFCA, for each sub-area, a path planning will be created according to the given parameters, then the system will show this path planning for the supervisor, the last step will be to select the set of trucks that will take this path planning and start the milk collection journey.

- Supervising and monitoring: During the daily collection, our system will show you a set of information about the status of each path planning and truck, using the web application, the supervisor can have access to the actual location of the truck and see the status report about each trip. They will be able to see the current quantity of milk transported and the estimated time to finish the trip. Also, the system provides you with a detailed report that contains all information about each trip from the start of the truck until the last visiting point inside the area of collection. All the trips and decisions taken during the process of the collection will be stored inside a DCA, this agent will store all that information and will be used to improve the process of collection and create new alternative solutions to improve the strategy of collection and driver behaviors. 


\section{Routing Agent}

The next step to do is to generate a set of path planning for trucks, each path will visit various milk units, so our main objective is to find the best way to compute and prepare this path planning based on the available data. To make this work, we apply a new distributed strategy based on ant colony optimization. In this strategy, we create various agents, one of them is the Worker Agent is dedicated to searching for the best route between a set of nodes based on various constraints such as the total distance between nodes and the quantity of milk inside each node, finally, the traffic flow at a specific area. The second entity is the Routing Agent, this one will take control of the proposed paths from each WA and will give them all the needed data to compute and find the best paths.

The basic concept of the ACO (Yang, H. X., \& Liu, T. 2013) is to simulate the ant as a truck that aims to visit a set of locations (cooperative) starting from the root node (dairy factory). In the beginning, each ant will start their journey by visiting a set of nodes, after each transition, a new amount of pheromone will be deposited on the ground to mark the way taken by this ant and to inform others with his path. The density of pheromone will allow other ants to evaluate the quality of the path followed by this ant, because more pheromone is produced on the ground it means that this path is the shortest path between the nest and food source.

So, in our distributed strategy we implement the same behavior, by replacing the real ants with autonomous agents, those agents will work in parallel to explore the environment and to compute the best paths from a start node to a destination node. So, our environment will be modeled as a graph $G=(F, R)$ made from a set of farms and routes. Each ant (agent) in the colony will try to begin his journey from a start node and will try to visit the next node by applying a probabilistic transition that takes in parameter the amount of pheromone between the two nodes and the coast of the route between the two adjacent nodes (in our case, it represents the linear distance):

$$
p_{i j}^{k}=\left\{\frac{\left[\tau_{i j}\right]^{\alpha}\left[\eta_{i j}\right]^{\beta}}{\sum_{h \in \Omega}\left[\tau_{i h}\right]^{\alpha}\left[\eta_{i h}\right]^{\beta}} 0 \quad \text { otherwise, if } j \in \Omega\right.
$$

As you can see, the probability $p_{i j}^{k}$ allows us to identify the probability that the agent $\mathrm{k}$ in the node (i) can choose to move toward the node (j) based on three constraints:

- We should verify that the node ( $\mathrm{j}$ ) are not already visited by the agent $\mathrm{k}$;

- We should take the amount of the previous deposited pheromone on this route;

- We consider the distance between the two nodes.

The parameters $[\beta, \alpha]$ are adjustable and they control the influence of the distance according to the amount of pheromone in the process of selecting the best next node. When an agent finished his tour (by visiting all nodes or by arriving at the end condition), it will be able to update the new amount of pheromone that will be applied to the set of visited routes. This new amount is computed according to the equation below:

$$
\tau_{i j}=(1-\rho) \cdot \tau_{i j}+\Delta \tau_{i j}
$$

The parameter $\Delta \tau_{i j}$ is calculated according to the total distance of the trip made by this agent, each one of the agents will update the amount of pheromone on the different routes used to compute the best path. So to calculate the new amount of pheromone we use the next equation: 
$\Delta \tau_{i j}=\sum_{n=1}^{N} \frac{Q}{L_{n}}$

The attribute $L_{n}$ represents the total distance of the trip made by the agent $\mathrm{n}$, the parameter $\mathrm{Q}$ is a heuristic value that can be between 0 and 1, is used only to have a control on the convergence of our algorithm.

After this process of pheromone update is done, each agent will share his best trip with the RA, those trips will be compared to extract only the best of them that respect our main constraints such us the total distance of the global trip, we need to minimize the total travelling time by avoiding traffic jam. As you can see in figure 8 , we have a set of worker agents that simulate the same behaviour of real ants, the try to find the shortest path, all the computed paths will be sent to the RA. The collected solutions will be communicated with the DFCA to give him a vision of each path and the set of nodes that can be visited with an approximation of the total distance and the average travelling time.

\section{SIMULATION AND RESULTS FOR THE DISTRIBUTED STRATEGY OF MILK-RUN}

To build our platform, we are using the Hadoop technology for preparing the environment, this technology will allow us to create a set of services (actors), each one of them will have a set of behaviours and roles to apply, the data collected will be analyzed and shared with those actors, finally, each one will give us a significant data that will be adopted in the milk transportation system. This distributed platform is divided into two sectors:

- Firstly, we need to collect all data from the different components and then store in specific databases, at this time, a new job is done to analyze that information to prepare the environment that will be adopted for preparing the path planning for each truck. So our environment will be viewed as a set of sub-graphs, each one contains a set of milk units that will be visited by specific

Figure 8. Distributed ACO based on autonomous agents RA and WA

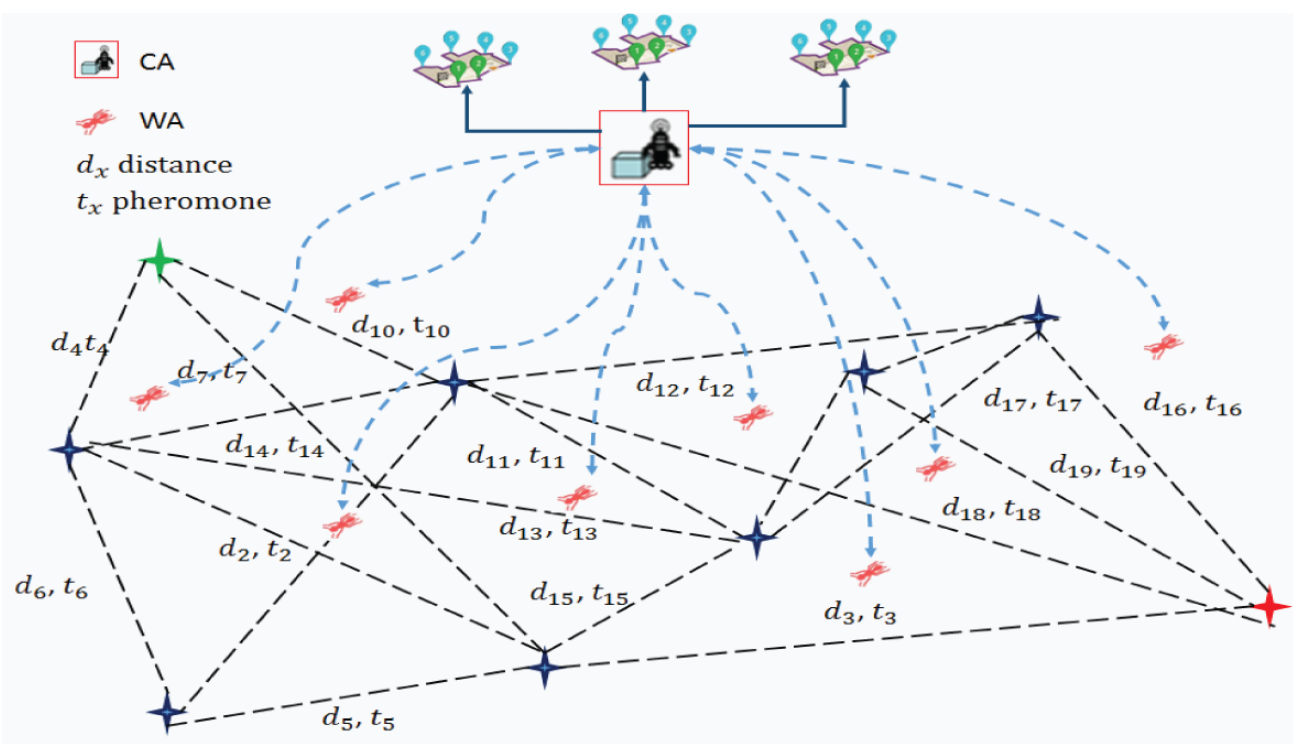


trucks. Also, the quantity of milk to pick-up will be known before preparing the path planning. This information is received from smart components attached to each unit of milk (farm).

- Secondly, when the process of collection is started, we allow the manager of the system to login into a specific web application to view the process of collection in real-time and to have access to a variety of information about each trip.

\section{Preparation Phase of the Distributed System for the Milk-Run}

Our web application will be developed based on the JavaScript and PHP 5 technologies to prepare the frontend, for storing data we use a MongoDB database which allows us to store unlimited data. Firstly, we need to give the manager of the system a sample interface for injecting data about farms (each farm is characterized by the name, code, latitude, longitude, road number, etc). That information will be used to draw our environment (map of the milk collection). To draw the final graph (Figure 9) we will use the services provided by Google Maps to draw routes between those units. The cost of each route will be the linear distance and the average travelling time. When the system computes the path planning for each truck, we use the same map to draw those paths planning. To find the shortest path we are based on the ACO strategy for this part. The coordination between the front-end and the back-end is done using custom web services developed for this type of communication.

Once the best solution is prepared, our platform will display the details about this trip (Figure 10) like the set of units to visit and the total distance of the trip and the average time consumed to finish the trip, also, the total quantity of milk that will be shipped. According to the total quantity of milk, our system will propose to the user a set of trucks according to the capacity of each one of them that can be selected for the process of collection, but the user can select his specific trucks.

To keep a real-time view about the process of milk collection, our system will allow the manager to visualize the details of each trip (Figure 11) such as the current position of the truck, the number of visited/un visited units and the quantity of milk not collected yet. Also, the manager can have a reporting option that helps him to export all data about each trip or vehicle to analyze the situation of each trip and to propose new decisions to improve the process of milk collection for a specific vehicle or an area.

\section{Validation Phase of the Simulation Model}

To show the effectiveness of our approach, we search for available data about the milk logistic in our country to identify the process of work and to find the strategy adopted in the traditional method

Figure 9. Wokflow of preparing a new mil run collection trip

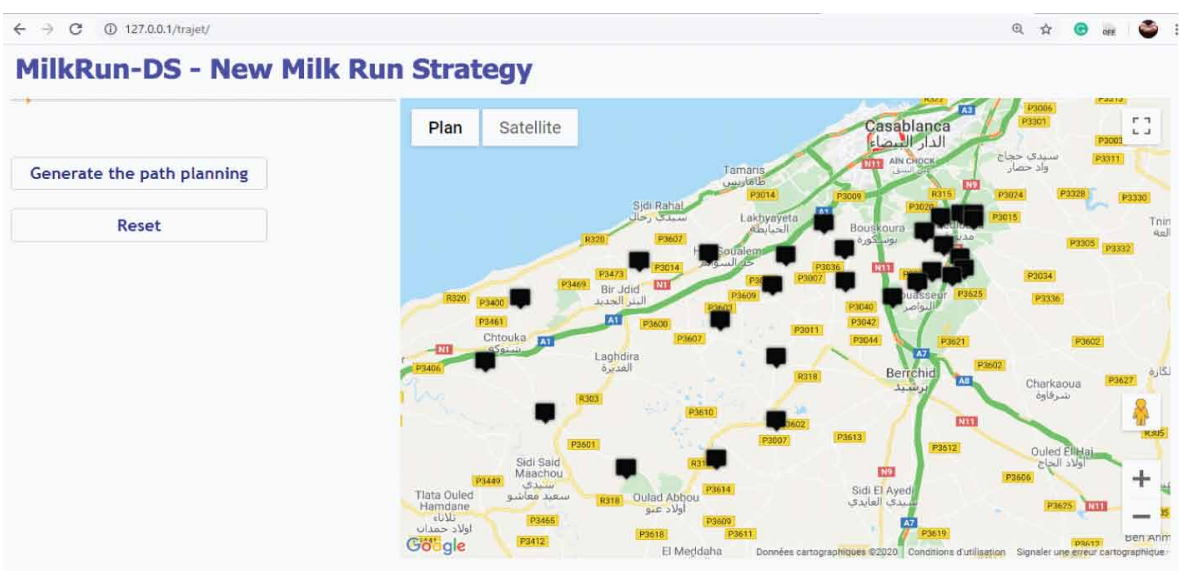


Figure 10. Best path planning for a given milk run workflow

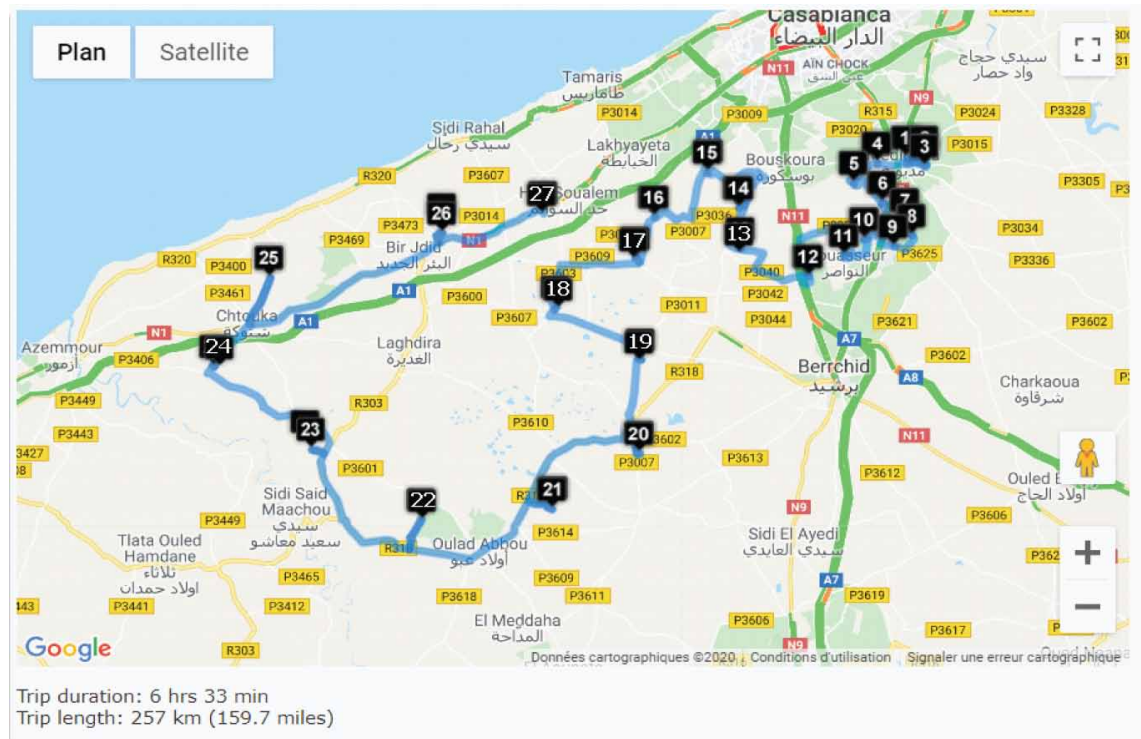

Figure 11. Overview of the status report of milk run trip

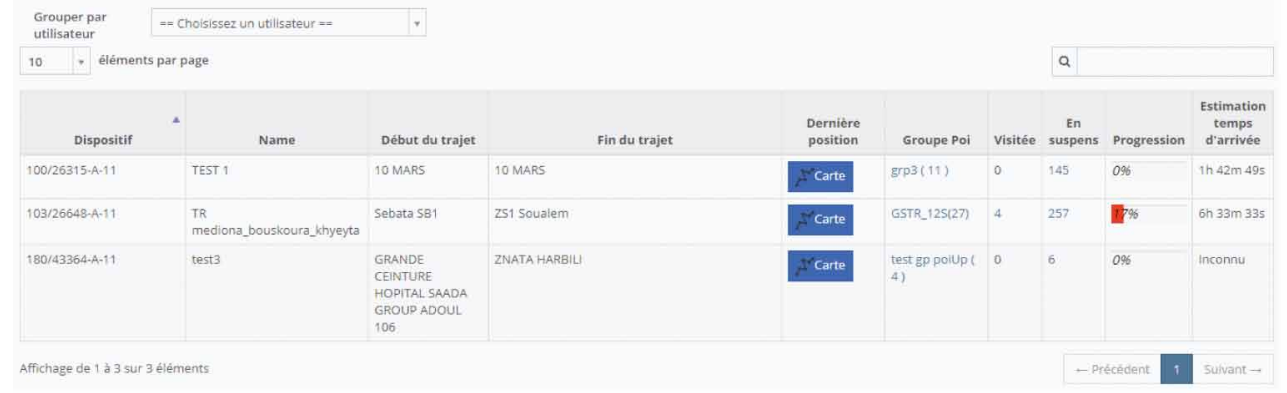

for milk collection. In Morocco, getting information is difficult because the access to information is restricted only for specific categories. But we prepared a dataset about two companies: Copag and Danone. This dataset contains the set of farms and cooperatives which are visited for the milk collection. Using this information, we can draw a geographical map that contains all those points of interest. As we can see that our main objective is to minimize the total distance of each trip to visit the set of milk units, maximize the total quantity of milk, and minimize the fuel consumed during the transportation of milk from farms toward the dairy factory. Using this dataset, we create a set of scenarios or bins (each one contains a set of farms to visit during a specific journey), we compare the process of collection between the classical strategy based on human decision and our distributed strategy based on the IoT technology and swarm intelligence for preparing the optimal routes.

As showing in table 1, we can see that the two methods or strategies are trying to give us the best solutions based on the available dataset. To compute the cost of transportation of each trip we need to get the total distance travelled and the amount of fuel consumed and the quantity of milk collected during this journey. As we can see, in the traditional strategy, the average total distance that can be done is about $164 \mathrm{~km}$ to visit a set of 16 farms with $7 \mathrm{~h}$ hours of traveling time to finish 
Table 1. Milk run distributed strategy versus traditional strategy: Case Danone and Copag

\begin{tabular}{|c|c|c|c|c|c|c|c|c|c|c|c|c|}
\hline \multirow[b]{2}{*}{$\begin{array}{c}\mathbf{N}^{\circ} \\
\text { Tournée }\end{array}$} & \multirow[b]{2}{*}{$\begin{array}{c}\text { Size } \\
\text { of } \\
\text { POIs }\end{array}$} & \multirow[b]{2}{*}{$\mathrm{Dh} / \mathrm{L}$} & \multicolumn{5}{|c|}{ Distributed Milk Run } & \multicolumn{5}{|c|}{ Classic Milk Run } \\
\hline & & & $\begin{array}{l}\text { Qty Milk } \\
\text { (L) }\end{array}$ & $\begin{array}{c}\text { Traveling } \\
\text { Time }\end{array}$ & $\begin{array}{c}\text { Distance } \\
(\mathbf{K m})\end{array}$ & $\begin{array}{l}\text { Fuel } \\
\text { used } \\
\text { (FU) }\end{array}$ & $\mathbf{L} / \mathbf{K m}$ & $\begin{array}{l}\text { Qty Milk } \\
\text { (L) }\end{array}$ & $\begin{array}{l}\text { Traveling } \\
\text { Time }\end{array}$ & $\begin{array}{l}\text { Distance } \\
(\mathbf{K m})\end{array}$ & $\begin{array}{l}\text { Fuel } \\
\text { used } \\
\text { (FU) }\end{array}$ & $\mathbf{L} / \mathbf{K m}$ \\
\hline 1 & 18 & 3,37 & 8088 & 06:09:44 & 191,49 & 74,68 & 42,24 & 7181 & $07: 18: 14$ & 255,89 & 99,80 & 28,06 \\
\hline 2 & 18 & 2,80 & 6413 & 05:06:16 & 136,71 & 53,32 & 46,91 & 5694 & 05:52:03 & 157,82 & 61,55 & 36,08 \\
\hline 3 & 16 & 3,21 & 3672 & $05: 42: 55$ & 211,76 & 82,59 & 17,34 & 3260 & 07:08:57 & 252,63 & 98,53 & 12,90 \\
\hline 4 & 18 & 2,44 & 8477 & 05:01:51 & 134,11 & 52,30 & 63,21 & 7526 & $05: 40: 48$ & 151,41 & 59,05 & 49,71 \\
\hline 5 & 16 & 2,98 & 5661 & $03: 41: 52$ & 126,20 & 49,22 & 44,86 & 5026 & $04: 10: 29$ & 142,49 & 55,57 & 35,27 \\
\hline 6 & 20 & 2,97 & 7313 & $05: 12: 33$ & 177,26 & 69,13 & 41,26 & 6493 & $07: 33: 42$ & 231,32 & 90,21 & 28,07 \\
\hline 7 & 15 & 2,82 & 7327 & 05:03:04 & 165,73 & 64,63 & 44,21 & 6505 & $06: 26: 35$ & 204,12 & 79,61 & 31,87 \\
\hline 8 & 22 & 3,50 & 4865 & $04: 55: 47$ & 128,06 & 49,94 & 37,99 & 4319 & $06: 37: 53$ & 173,99 & 67,85 & 24,82 \\
\hline 9 & 18 & 2,54 & 6285 & 05:09:04 & 118,30 & 46,14 & 53,13 & 5580 & $06: 18: 52$ & 150,99 & 58,89 & 36,96 \\
\hline 10 & 15 & 3,44 & 6449 & 05:08:08 & 182,37 & 71,13 & 35,36 & 5726 & $05: 52: 40$ & 209,27 & 81,61 & 27,36 \\
\hline 11 & 11 & 2,45 & 6006 & $03: 19: 27$ & 106,30 & 41,46 & 56,50 & 5333 & $04: 45: 28$ & 128,10 & 49,96 & 41,63 \\
\hline 12 & 18 & 1,97 & 10858 & $05: 11: 50$ & 139,22 & 54,30 & 77,99 & 9640 & $06: 37: 30$ & 177,87 & 69,37 & 54,20 \\
\hline 13 & 14 & 2,19 & 8833 & $04: 23: 56$ & 123,32 & 48,09 & 71,63 & 7843 & 05:01:05 & 140,91 & 54,95 & 55,66 \\
\hline 14 & 21 & 1,81 & 12536 & $06: 46: 46$ & 150,57 & 58,72 & 83,26 & 11130 & 07:39:16 & 170,00 & 66,30 & 65,47 \\
\hline 15 & 23 & 1,65 & 11828 & $05: 37: 42$ & 124,62 & 48,60 & 94,91 & 10502 & $06: 21: 17$ & 140,70 & 54,87 & 74,64 \\
\hline 16 & 17 & 2,19 & 6951 & $05: 19: 32$ & 111,32 & 43,42 & 62,44 & 6172 & $06: 15: 20$ & 131,78 & 51,39 & 46,84 \\
\hline 17 & 21 & 2,32 & 12823 & $07: 16: 47$ & 216,78 & 84,55 & 59,15 & 11385 & $08: 24: 03$ & 250,22 & 97,58 & 45,50 \\
\hline 18 & 19 & 2,50 & 12034 & $06: 47: 12$ & 218,83 & 85,34 & 54,99 & 10684 & 07:59:52 & 258,41 & 100,78 & 41,35 \\
\hline 19 & 11 & 1,82 & 8549 & $03: 46: 32$ & 95,51 & 37,25 & 89,51 & 7590 & $05: 41: 24$ & 122,75 & 47,87 & 61,84 \\
\hline 20 & 5 & 2,67 & 5550 & 02:57:01 & 107,42 & 41,89 & 51,67 & 4927 & 04:24:01 & 124,11 & 48,40 & 39,70 \\
\hline 21 & 26 & 1,03 & 13649 & 05:10:41 & 84,54 & 32,97 & 161,46 & 12119 & 06:08:04 & 113,09 & 44,10 & 107,17 \\
\hline 22 & 25 & 1,33 & 11819 & $05: 21: 07$ & 96,26 & 37,54 & 122,79 & 10493 & $06: 16: 48$ & 124,22 & 48,44 & 84,47 \\
\hline 23 & 26 & 1,72 & 11704 & $06: 21: 16$ & 129,55 & 50,52 & 90,34 & 10392 & $07: 34: 26$ & 156,45 & 61,02 & 66,42 \\
\hline 24 & 27 & 1,46 & 12338 & 06:05:19 & 112,81 & 44,00 & 109,37 & 10954 & 07:05:22 & 136,19 & 53,11 & 80,43 \\
\hline 25 & 24 & 1,26 & 9382 & $04: 57: 18$ & 69,19 & 26,98 & 135,59 & 8330 & $06: 15: 34$ & 103,64 & 40,42 & 80,38 \\
\hline 26 & 22 & 1,63 & 9536 & $05: 00: 14$ & 95,33 & 37,18 & 100,04 & 8467 & $07: 52: 38$ & 119,60 & 46,64 & 70,80 \\
\hline 27 & 13 & 2,45 & 5419 & 04:03:46 & 93,93 & 36,63 & 57,69 & 4811 & 05:08:42 & 124,53 & 48,57 & 38,63 \\
\hline 28 & 23 & 1,17 & 13260 & 04:30:04 & 95,14 & 37,10 & 139,38 & 11773 & $05: 22: 10$ & 121,80 & 47,50 & 96,66 \\
\hline 29 & 29 & 3,12 & 9306 & 08:05:46 & 208,51 & 81,32 & 44,63 & 8263 & $09: 24: 51$ & 246,12 & 95,99 & 33,57 \\
\hline 30 & 18 & 1,25 & 12737 & $04: 05: 25$ & 97,28 & 37,94 & 130,93 & 11308 & $04: 46: 36$ & 116,34 & 45,37 & 97,20 \\
\hline AVG & 18 & 2,27 & 8988,93 & $5: 12: 38$ & 134,95 & 52,63 & 74,03 & 7980,87 & 6:24:09 & 164,56 & 64,18 & 53,12 \\
\hline
\end{tabular}

the trip. But this journey will only give us an average of 8000 liters that can be collected during this journey. Compared with the distributed strategy, our truck fleet can collect an average of 9000 liters only on $135 \mathrm{~km}$ and with $5 \mathrm{~h}$ hours of work. This means that our strategy tries to find the shortest path between the set of farms using the ACO strategy which helps us to compute the best routes based on the linear distance between farms. When we minimize the total distance of the trip that means the total fuel consumed will be decreased in parallel. By the way, the cost of the trip will be minimized according to the fuel consumed and the quantity of milk transported.

As showing in this table, the process of working in parallel helps our system to find the shortest paths. this behavior is based on the presented multi-agent architecture which contains a set of agents, 
also the clustering strategy gives us more flexibility to split our main problem into a set of subproblems. After that, we can create a set of colonies to explore this sub-graph and prepare the best paths for each one of them.

The result of this simulation approves that our distributed strategy will be a good tool for milk logistics if you went to optimize the cost of the trip and increase the quantity of milk transported. This work aims to use a distributed strategy of the ACO combined with the IoT technology to make a new distributed decision system that controls and help users to optimize the problems related to the milk collection schedule. The results shown in this simulation approve the quality of our solution and we will try to apply our distributed strategy on various datasets to improve the quality of solutions made by our distributed system for milk run transportation.

\section{CONCLUSION}

The aim of Milk-Run's logistics system is to reduce the number of trucks for milk collection in a specific area. Actually, with the emergence of IoT technology, Big Data and swarm intelligence, this work can be done by combining those technologies to find and compute the best solutions for this system based on various constraints injected by humans. In this work, we introduced a distributed strategy for the milk system by preparing a set of path planning for trucks based on a network of communication between farms and trucks. This system gives us an idea about the quantity of milk available on each farm based on IoT technology. For the process of path planning, we are using the ACO strategy to compute and find the shortest path between those farms. The whole system is running in parallel, this mechanism allows us to minimize the process of communication between actors and maximize the productivity of our system to give good result for each specific scenario. 


\section{REFERENCES}

Bocewicz, G., Nielsen, P., \& Banaszak, Z. (2019). Declarative modeling of a milk-run vehicle routing problem for split and merge supply streams scheduling. In Advances in Intelligent Systems and Computing (Vol. 853, pp. 157-172). Springer Verlag. doi:10.1007/978-3-319-99996-8_15

Caria, M., Todde, G., \& Pazzona, A. (2018). Modelling the collection and delivery of sheep milk: A tool to optimise the logistics costs of cheese factories. Agriculture (Switzerland), 8(1), 5. Advance online publication. doi:10.3390/agriculture 8010005

Chen, Z., \& Sarker, B. R. (2014). An integrated optimal inventory lot-sizing and vehicle-routing model for a multisupplier single-assembler system with JIT delivery. International Journal of Production Research, 52(17), 5086-5114. doi:10.1080/00207543.2014.899715

Dat Minh, N., Trung Kien, D., \& Khac Hau, P. (2020). Applying milk-run method to optimize cost of transport: An empirical evidence. International Journal of Supply and Operations Management, 7(2), 178-188. doi:10.22034/ IJSOM.2020.2.6

Hfeda, M., Marchand, F., \& Dao, T.-M. (2017). Optimization of milk-run delivery issue in lean supply chain management by genetic algorithm and hybridization of genetic algorithm with ant colony optimization: An automobile industry case study. Journal of Management and Engineering Integration, 10(2), 90-99.

Jafari-Eskandari, M., Sadjadi, S. J., Jabalameli, M. S., \& Bozorgi-Amiri, A. (2009). A robust optimization approach for the Milk Run problem (An auto industry supply chain case study). International Conference on Computers \& Industrial Engineering, 1076-1081. doi:10.1109/ICCIE.2009.5223541

Ji-li, K., Guo-zhu, J., \& Cui-ying, G. (2013). A new mathematical model of vehicle routing problem based on milk-run. 2013 International Conference on Management Science and Engineering 20th Annual Conference Proceedings, 385-392. doi:10.1109/ICMSE.2013.6586310

Kale, S. (2020). Milk run technique to ensure effective just in time technique practices. International Journal of Advanced Science and Technology, 29(8), 1302-1307.

Klenk, E., Galka, S., \& Günthner, W. A. (2015). Operating Strategies for In-Plant Milk-Run Systems. IFACPapersOnLine, 48(3), 1882-1887. 10.1016/j.ifacol.2015.06.361

Kong, J. L., Jia, G. Z., \& Gan, C. Y. (2013). A new mathematical model of vehicle routing problem based on milk-run. In International Conference on Management Science and Engineering - Annual Conference Proceedings (pp. 385-392). doi:10.1109/ICMSE.2013.6586310

Kothandaraman, D., Sheshikala, M., Seena Naik, K., Chanti, Y., \& Vijaykumar, B. (2019). Design of an optimized multicast routing algorithm for internet of things. International Journal of Recent Technology and Engineering, 8(2), 4048-4053. doi:10.35940/ijrte.B3372.078219

Ma, J., \& Sun, G. (2013). Mutation ant colony algorithm of milk-run vehicle routing problem with fastest completion time based on dynamic optimization. Discrete Dynamics in Nature and Society, 2013, 1-6. Advance online publication. doi:10.1155/2013/418436

Mouhcine, E., Khalifa, M., \& Mohamed, Y. (2020). Optimized Path Planning for Electric Vehicle Routing and Charging Station Navigation Systems, Special Issue Submission: Intelligent Optimization and Its Application Applied Metaheuristic Computing. International Journal of Applied Metaheuristic Computing, 11(3), 3. Advance online publication. doi:10.4018/IJAMC.20200701

Mouhcine, E., Yassine, K., Hanaa, E. F., Mansouri, K., \& Mohamed, Y. (2019). An Internet of Things (IOT) based Smart Parking Routing System for Smart Cities. International Journal of Advanced Computer Science and Applications, 10(8). Advance online publication. doi:10.14569/IJACSA.2019.0100870

Nguyen, T.-H.-D., \& Dao, T.-M. (2015). Supply chain milk-run delivery optimization. Journal of Management and Engineering Integration, 8(1), 29-40.

Qu, T., Chen, Y. D., Wang, Z. Z., Nie, D. X., Luo, H., \& Huang, G. Q. (2015). Internet-of-Things-based justin-Time milk-run logistics routing system. In ICNSC 2015 - 2015 IEEE 12th International Conference on Networking, Sensing and Control (pp. 258-263). Institute of Electrical and Electronics Engineers Inc. doi:10.1109/ ICNSC.2015.7116045 
Sestino, A., Prete, M. I., Piper, L., \& Guido, G. (2020). Internet of Things and Big Data as enablers for business digitalization strategies. Technovation, 98. 10.1016/j.technovation.2020.102173

Starzec, M., Starzec, G., Byrski, A., Turek, W., \& Piętak, K. (2020). Desynchronization in distributed Ant Colony Optimization in HPC environment. Future Generation Computer Systems, 109, 125-133. doi:10.1016/j. future.2020.03.045

Yang, H. X., \& Liu, T. (2013). Optimization and simulation of express's vehicle routing in commercial district based on ant colony algorithm. Information Technology Journal, 12(24), 8462-8468. doi:10.3923/ itj.2013.8462.8468

Zhang, H., \& Lu, X. (2020). Vehicle communication network in intelligent transportation system based on Internet of Things. Computer Communications, 160, 799-806. doi:10.1016/j.comcom.2020.03.041 